Décadrages Décadrages

cınéma, à travers champs Cinéma, à travers champs

39 | 2018

Jeu vidéo et cinéma

\title{
Les régimes effrayants de la vision vidéoludique*
}

\section{Bernard Perron}

\section{(2) OpenEdition}

\section{Journals}

Édition électronique

URL : https://journals.openedition.org/decadrages/1316

DOI : 10.4000/decadrages.1316

ISSN : 2297-5977

\section{Éditeur}

Association Décadrages

Édition imprimée

Date de publication : 1 décembre 2018

Pagination : 8-29

ISBN : 978-2-9700963-5-7

ISSN : 2235-7823

\section{Référence électronique}

Bernard Perron, "Les régimes effrayants de la vision vidéoludique* », Décadrages [En ligne], 39 | 2018, mis en ligne le 05 février 2021, consulté le 04 avril 2022. URL : http://journals.openedition.org/ decadrages/1316 ; DOI : https://doi.org/10.4000/decadrages.1316

(B) Décadrages 
* Cet article est une version abrégée du chapitre sept de mon ouvrage The World of Scary Video Game: A Study in Videoludic Horror, New York, Bloomsbury, 20I8. Toutes les citations en anglais sont librement traduites par l'auteur.

1 Peter Hutchings, Historical Dictionary of Horror Cinema, Lanham, Scarecrow Press, 2008, p. II2.

2 Par exemple, des yeux effrayés sont au centre de la jaquette du jeu Haunted House pour l'Atari 2600 (I98r), tandis que ce motif est accompagné de chaînes et de mains sur la bouche sur celles de Condemned: Criminal Origins (Monolith Productions, 2005) et Rise of Nightmares (Sega, 20II). Le «T» dans le titre de The Evil Within (Tango Gameworks, 20I4) forme une pointe dirigée vers l'œil du visage d'un homme enveloppé de barbelés. L'extrême gros plan d'un œil infecté constitue l'image de couverture de Resident Evil: Revelation 2 (Capcom, 20I4).
Bernard Perron

\section{Les régimes effrayants de la vision vidéoludique *}

LES FICTIONS D'HORREUR ONT TOUJOURS POUSSÉ - et la plupart du temps forcé - des personnages à voir l'invisible, à regarder l'insupportable, à découvrir des menaces imminentes pour leur santé physique et mentale ainsi qu'à affronter des entités monstrueuses et des dangers effroyables pour l'humanité. C’est ce qui définit selon Peter Hutchings ce genre cinématographique:

\section{«A bien des égards, l'œil est le principal organe humain du cinéma d'hor- reur. Les réalisateurs utilisent fréquemment des gros plans des yeux des victimes, grands ouverts et sans défense, et ceux des monstres, rétrécis et agressifs, pour accentuer les sensations sado-masochistes du genre.» ${ }^{1}$}

En se référant à l'œil tranché par un rasoir dans Un chien andalou de Luis Bunũel (1928), Hutchings souligne également que les yeux ont été l'objet des moments les plus violents du genre de l'horreur. Si des images de globes oculaires effrayants et d'expressions de frayeur peuvent être trouvées sur la couverture de boîtes de jeux vidéo d'épouvante ${ }^{2}$, ceuxci - à la différence du cinéma d'horreur - ne se concentrent pas sur l'œil, mais plutôt sur le point de vue, au sens figuré et littéral. Bien que la peur soit toujours médiatisée suivant des codes culturels, elle reste une affaire personnelle, une question de point de vue. Puisque les mondes de jeu peuvent être montrés à partir de différentes perspectives, cet article analyse les tactiques de l'épouvante basées sur le point de vue, qui compliquent l'action et la vision du joueur afin de susciter la peur.

\section{Point de vue et point de (ré)action}

Dans Understanding Video Games: the Essential Introduction, Simon Egenfeldt-Nielsen, Jonas Heide Smith et Susana Pajares Tosca différencient les deux points de vue majoritairement proposés dans le jeu vidéo qui sont au cœur de la présente étude:

«Dans la perspective à la première personne, nous voyons l'action du point de vue du protagoniste - comme si le joueur lui-même vivait 
l'action. Dans la perspective à la troisième personne, le joueur regarde tout ce qu'il contrôle, ce qui peut correspondre à un objet à l'écran (un personnage, un véhicule), à un certain nombre d’objets (une armée de soldats, un groupe de villageois) ou à différents paramètres (tels que les taux d'imposition ou le zonage de la ville).» ${ }^{3}$

Il est certes contraire à l'usage commun de se référer à la perspective à la troisième personne pour décrire une vue sur les unités dans un jeu de stratégie en temps réel comme Starcraft (Blizzard Entertainment, I998) ou les différents niveaux influençant la zone urbaine peuplée de SimCity (Maxis Software, 1989) - c'est l'exemple donné dans Understanding Video Games ${ }^{4}$. Il s'agit plutôt de perspectives isométriques et aériennes. De plus, Egenfeldt-Nielsen et al. utilisent le travail du concepteur Will Wright pour illustrer le fait que «nous pouvons profiter de l'interaction avec un système sans avoir besoin de nous identifier à des personnages concrets (processus au centre du film narratif, par exemple)» ${ }^{5}$. Dans le cadre d'une réflexion sur le jeu vidéo d'épouvante, une telle affirmation souligne en retour que la peur vidéoludique est très difficile à susciter dans un système de jeu plus abstrait - à moins que le système ne soit luimême vu comme diabolique et malveillant. Un joueur peut être stressé par une série d'événements qui stoppent la croissance de sa ville ou être énervé par des catastrophes qui détruisent ses méga-tours, mais il n’a pas peur pour autant. Une situation dans un jeu sera considérée comme effrayante si elle est jugée dangereuse pour quelqu'un, que ce soit un personnage non-joueur (ou plusieurs s'ils ont une individualité) ou un personnage-joueur (visible ou non à l'écran).

Britta Neitzel propose une distinction très pertinente pour mieux analyser le jeu vidéo:

«On peut définir les jeux informatiques comme un processus d'autoobservation avec une rétroaction continue. Dans ce processus, la position du joueur est double. En plus de sa position d'agent dans le jeu, on lui attribue également la position d'un observateur, qui à son tour détermine sa position en tant qu'agent dans l'espace visuel. [...] Pour éviter une prolifération de la terminologie, jutiliserai le terme point d'action pour la position active et celui de point de vue pour la position d'observation.» ${ }^{6}$

Neitzel adapte le concept narratologique de la focalisation (qui voit?) afin de renvoyer à un point de vue subjectif pour la perspective à la première personne. La perspective à la troisième personne est quant à elle
3 Simon Egenfeldt-Nielsen, Jonas Heide Smith et Susana Pajares Tosca, Understanding Video Games: The Essential Introduction, New York, Routledge, 2008, p. 107.

4 Et si, comme beaucoup de commentaires l'ont suggéré, SimCity fait en sorte que le joueur se considère comme un dieu face à la ville ou comme le maire, nous aurions alors droit à une perspective à la première (et non à la troisième) personne.

5 Simon Egenfeldt-Nielsen, Jonas Heide Smith et Susana Pajares Tosca, op.cit., p. 107 .

6 Britta Neitzel, «Narrativity in Computer Games», dans Joost Raessens et Jeffrey Goldstein (éd.), Handbook of Computer Games Studies, Cambridge, MIT Press, 2005, pp. 230-23I. 
7 Puisque les jeux vidéo ont été programmés et que les concepteurs tentent souvent de prédire les réactions des joueurs en développant des jeux qui réagissent aux réponses de ces derniers (en partie par l'entremise de la programmation d'intelligence artificielle), Arsenault et moi-même avons souligné que le jeu vidéo serait finalement peut-être mieux compris en termes d'inter(ré)activité plus que d'interactivité: «[N]ous ferons valoir qu'un jeu vidéo est plutôt une chaîne de réactions. Le joueur n'agit pas autant qu'il réagit à ce que le jeu lui présente, et de même, le jeu réagit à son tour à sa réponse». Dominic Arsenault et Bernard Perron, «In the Frame of the Magic Cycle: the Circle(s) of Gameplay», dans Bernard Perron et Mark J.P. Wolf (éd.), The Video Game Theory Reader 2, New York, Routledge, 2009, pp. II9-I20.

8 Brenda Laurel, Computers as Theatre, Boston, Addison-Wesley, I99I, p. II7.

9 Janet H. Murray, Hamlet on the Holodeck: The Future of Narrative in Cyberspace, New York, Free Press, I997, p. I26. divisée entre un point de vue semi-subjectif lorsque la caméra suit un personnage, comme dans Tomb Raider (Core Design, I996), et un point de vue objectif lorsque l'univers est présenté de l'extérieur. Le point d'action est décrit en posant les questions suivantes: Est-il situé à l'intérieur ou à l'extérieur du monde du jeu (intra ou extra-diégétique)? Est-il situé ou non au centre de l'écran (concentrique ou excentrique)? Est-ce que cela conduit à des résultats instantanés ou non (directs ou indirects)? J'y reviendrai, mais je noterai pour l'instant ce que Nietzel ne souligne pas: à savoir que le point de vue et le point d'action se chevauchent dans la perspective à la première personne, et qu'ils restent distincts à la troisième personne. A la lumière de ce modèle, SimCity fournit des points d'action qui sont extra-diégétiques (si l'on dit que l'on interagit avec un système), à la fois directs et indirects (les résultats des actions ne sont pas toujours immédiats), ainsi que multiples et excentriques (partout sur la carte). Au contraire, par l'intermédiaire du personnage-joueur, un jeu d'épouvante comme Resident Evil (Capcom, 1996) possède un point d'action qui est complètement identifié et localisé. C'est un point de (ré)action direct, intra-diégétique et centré - le joueur réagit aux menaces tandis que ces dernières se déroulent autour de lui ${ }^{7}$.

La notion de point d'action est fort instructive pour définir les principes de l'horreur vidéoludique. A la suite de Brenda Laurel ${ }^{8}$, Janet Murray a placé l'agentivité (agency) - à savoir «le pouvoir satisfaisant de prendre des mesures significatives et de voir les résultats de nos décisions et de nos choix ${ }^{9}{ }^{-}$au cour des plaisirs caractérisant les environnements numériques. Un joueur peut ressentir ce plaisir autant en construisant et en gérant sa ville dans SimCity qu'en déplaçant Jill Valentine dans les corridors du manoir de Resident Evil. Cependant, l'agentivité ne suffit pas à instiller un sentiment d'effroi. Conformément aux conclusions de King et Krzywinska, il est nécessaire de tenir compte de la sensation de présence:

«Les distinctions entre les degrés de présence sont étroitement corrélées avec les différences dans la perspective visuelle fournies sur le monde du jeu. Les jeux les plus distanciés ont tendance à être ceux qui utilisent des perspectives aériennes semblables à Dieu. La plus grande sensation de présence, ou d'immersion sensorielle dans le paysage de jeu, est généralement fournie par des jeux qui offrent la perspective à la première personne d'une figure située dans le monde fictif du jeu. Entre 
les deux, il y a les jeux qui offrent une variété de vues à la troisième personne, situées à l'intérieur du monde du jeu, mais pas directement à travers les yeux du personnage-joueur.» ${ }^{10}$

Le sentiment d'«être là» est certes un état ressenti à la première personne, mais moins en termes visuels que psychologiques. Les auteurs de Tomb Raiders \& Space Invaders illustrent cette participation par une référence à l'horreur:

«Le spectateur impliqué psychologiquement dans un film d'horreur «n’imagine pas qu'il a peur; il imagine avoir peur, et il l'imagine de l'intérieur».»"11

Un joueur sera d'autant plus effrayé si le point de (ré)action est centré et intra-diégetique; peu importe en revanche comment il est incarné. Pour cette raison, King et Krzywinska soulignent une corrélation fondamentale:

«La perspective à la première personne (présentée) ou à la troisième personne (représentée) est, dans cette considération [de la fantaisie], accessoire au degré d’implication vécu dans un scénario donné: ‘ Nous entrons dans la structure fantasmatique et nous nous identifions comme si c'était la nôtre ».» ${ }^{12}$

L'opposition entre les points de vue à la première et à la troisième personne n'est pas impérative pour faire l'expérience de la présence à l'intérieur du monde d'un jeu. Un joueur se préoccupe de lui-même, peu importe s'il contrôle le point de vue subjectif d'un protagoniste ou d'un personnage-joueur visible à l'écran. La spécificité de l'expérience repose néanmoins sur le point de vue à travers lequel le monde du jeu est vu et sur le point d'action à partir duquel un joueur interagit au sein de ce dernier.

Pour éviter de parler du seul aspect visuel du monde du jeu, j’emprunte à Steven Shaviro la notion de régime affectif de vision ${ }^{13}$. Puisque l'approche de Shaviro est ancrée dans le corps et dans les manières dont l'image assaille et agite un spectateur, elle reste en accord avec une étude des effets de l'horreur et de la peur vidéoludiques. Elle apparaît encore plus pertinente quand l'on sait qu'elle repose sur une analyse de Strange Days de Kathryn Bigelow (I995). Cette œuvre de science-fiction tourne autour du SQUID, un dispositif d'interférence quantique supraconductrice (Superconducting Quantum Interference Device) qui enregistre des informations provenant du cortex central et permet aux utilisateurs de
10 Geoff King et Tanya Krzywinska, Tomb Raiders \& Space Invaders. Videogames Forms and Contexts, Londres, I.B. Tauris, 2006 , p. 97.

11 Id., p. I2I. Les auteurs citent un passage de Kendall Walton, Mimesis as Make-Believe: On the Foundations of the Representational Arts, Cambridge, Harvard University Press, I990.

12 Id., p. I22. Ici, les auteurs citent un passage de Elizabeth Cowie, Representing the Woman: Cinema and Psychoanalysis, Londres, Macmillan Press, I997.

13 Steven Shaviro, "Regimes of Vision: Kathryn Bigelow, Strange Days », Polygraph, $\mathrm{n}^{\circ} \mathrm{I3}$, 2001, pp.59-68. En fait, je suis venu à Shaviro par l'entremise d'Alexander Galloway. Dans sa recherche des «origines du jeu de tir à la première personne», ce dernier se réfère succinctement au «régime affectif de vision» de Shaviro pour montrer comment la prise de vue subjective du film est devenue une vision centrale de l'espace vidéoludique. Voir Alexander R. Galloway, Gaming: Essays on Algorithmic Culture, Minneapolis, University of Minnesota Press, 2006, pp. 62-69. 
14 Steven Shaviro, "Regimes of Vision: Kathryn Bigelow, Strange Days», op. cit., p. 62.

15 Id., p. 67. vivre un événement à partir de la vision et des sensations de la personne qui les a enregistrées. Cela est présenté d'emblée au début du film par le point de vue subjectif et instable d'un homme qui braque un restaurant avec deux complices. La police arrivant rapidement sur les lieux, les deux hommes vont sur le toit. Alors que son ami réussit à sauter sur l'autre bâtiment, l'homme qui porte l'appareil n'atteint que le bord et tombe au moment où son partenaire le lâche pour éviter les tirs des policiers. La scène se termine lorsque l'homme touche le sol. Shaviro décrit cette séquence d'ouverture et l'explique en ces termes:

\footnotetext{
«Bigelow présente les enregistrements de SQUID comme des plans continus à la première personne. Les événements se déroulent en temps réel, en une seule prise, et d'un seul point de vue. Ces séquences sont tactiles, ou haptiques, plutôt que visuelles. La caméra subjective ne regarde pas seulement une scène. Elle se déplace activement à travers l'espace. Ça se bouscule, ça s’arrête et ça recommence, ça tourne et s’incline, ça se penche en avant et en arrière. Ça suit les rythmes du corps entier, pas seulement celui des yeux. C'est un régime de vision présubjectif, affectif et non cognitif.» ${ }^{\mathbf{1 4}}$
}

On comprend que les scènes de SQUID ressemblent à des jeux à la première personne, et notamment à un jeu de tir (First-Person Shooter) durant les premières minutes du film. Mais Strange Days n'étant pas seulement filmé avec le SQUID, Shaviro parle aussi de la manière dont la «vision est brisée et déshumanisée» dans les autres parties du film.

«Le deuxième régime de vision [...] n'identifie pas étroitement le specta-
teur avec un protagoniste, ni ne présente le genre d'objectivité à la troi-
sième personne auquel le style bazinien des plans-séquences nous fait
généralement penser. Au lieu de cela, la caméra semble créer un nouveau
point de vue, ni précisément objectif ni conventionnellement subjectif,
mais transpersonnel et social.» ${ }^{15}$

Bien que le parallèle soit moins explicite et qu'il doive être adapté à la configuration des jeux d'action-aventure généralement centrés sur un seul personnage, il s'applique tout de même au travail de la caméra des jeux à la troisième personne. Je reviendrai sur ces observations pour examiner les deux régimes de vision des jeux vidéo d'épouvante. Mais je vais d'abord proposer une analyse du régime à la troisième personne afin d'introduire une notion qui concerne les deux modalités de vision qui m’intéressent dans le cadre de la présente étude. 


\section{Le régime effrayant de la vision à la troisième personne}

Le cinéma ne manque pas d'être mentionné lorsque l'on parle de jeux vidéo d'épouvante. Au-delà de l'adaptation vidéoludique de Jaws de Steven Spielberg en 1975 (Shark Jaws, Horror Games et Atari, I975), d'un slasher comme Halloween (1978) de John Carpenter (même titre par Wizard Video Games, I983) et des films Alien dans les années I980, Paul Norman, le concepteur de Forbidden Forest (Cosmi, 1983), avait déjà voulu faire de son jeu un film ${ }^{16}$. C'est ce que les créateurs de Projet Firestart (Dynamix) ont revendiqué et annoncé ouvertement en I988 - on lit sur la jaquette arrière: «Plus qu'un jeu... un film d'horreur dans l'espace». Ensuite, par le biais de la perspective à la troisième personne et des angles de caméra prédéterminés, la cristallisation du survival horror a été actualisée par rapport au cinéma. Rappelons l'intention originale de Shinji Mikami, concepteur du premier opus de la franchise de Capcom:

«Je veux que Resident Evil donne au joueur le sentiment qu'il est le personnage principal d'un film d'horreur.» ${ }^{17}$

Effectivement, les concepteurs de jeux se sont approprié le langage cinématographique et ont utilisé avec grand succès les trucs du cinéma d'horreur. Cela dit, si dans un film le cadrage travaille la surface de l'image, détermine la manière dont les éléments seront vus, induit le mouvement des personnages suivant la profondeur du champ et implique un espace à l'extérieur de ses limites, dans un jeu il doit répondre avant tout aux besoins de la jouabilité, aux «contraintes ludiques». Les I7o plans de caméra d'Alone in the Dark conçu par Frédérick Raynal (I-Motion Inc. et Infogrames, I992) sont variés, mais restent jouables. Ce qui est articulé, c'est la surface de jeu, l'espace au sein duquel le joueur doit pouvoir agir. Et cette agentivité va modifier les aboutissants de la vision ${ }^{18}$.

Comme le notait André Bazin dans un essai très célèbre sur le théâtre et le cinéma:

«L'écran n'est pas un cadre comme celui du tableau, mais un cache qui ne laisse percevoir qu’une partie de l'événement. [...] L'écran n’a pas de coulisses [...]. A l'opposé de celui de la scène, l'espace à l'écran est centrifuge. ${ }^{19}$

L'horreur exploite à tous les égards ce que Pascal Bonitzer a si justement appelé le champ aveugle:
16 Dans le «Making of» de Forbidden Forest du magazine Retro Gamer, on peut lire: « Les gars dans la compagnie voulaient simplement un jeu de cibles avec un arc et des flèches ', se souvient Paul. 〈Je ne m'intéressais pas à ça - je suis cinéphile, pas un joueur, et il n'y a pas de film dans cette idée.) Par conséquent, Paul a tout fait pour que le jeu s'apparente à un film - d'où la forêt, les monstres, la musique et l'éclairage». Voir Craig Grannell, «The Making of Forbidden Forest», Retro Gamer, $\mathrm{n}^{\circ} 25$, mai 2006, p. $7 \mathrm{I}$.

17 The Feature Creature, «Creating Evil Incarnate: The Making of Resident Evil», GamePro, n ${ }^{9}$, avril I996, p. 33.

18 La réflexion résumée et modifiée ici est plus détaillée dans Bernard Perron, «Effrayantes aventures dans le champ aveugle du jeu vidéo et du cinéma d'horreur», dans Alexis Blanchet (éd.), Cinéma et jeux vidéo, Paris, Questions Théoriques, 20I7, Pp. 5-I5.

19 André Bazin, «Théâtre et cinéma» (195I), Qu'est-ce que le cinéma?, Paris, Editions du Cerf, coll. $7^{\mathrm{e}}$ art, I985, p. I6o. 
20 Pascal Bonitzer, «Le champ aveugle», Le Champ aveugle. Essais sur le cinéma, Paris, Cahiers du cinéma et Gallimard, I982, p. 96.

21 Id., p. 97.

22 Pascal Bonitzer, «La vision partielle» [I979], La Vision partielle. Ecrits sur le cinéma, Paris, Capricci, 20ı6, p. 204.
«Le champ visuel, au cinéma, se dédouble... d’un champ aveugle. L'écran est un cache et la vision partielle. Le cinéma en l'occurrence raffine ou renchérit sur un effet qui ne lui est pas absolument spécifique [...]. L'ennemi est virtuellement partout si la vision est partielle.» 20

Qui plus est, «le cinéma veut [...] que ce qui a lieu dans la contiguïté du hors-champ ait autant d'importance, du point de vue dramatique - et même parfois davantage - que ce qui a lieu à l'intérieur du cadre. C'est tout le champ visuel qui est dramatisé [...]» ${ }^{21}$. Dans un autre essai, Bonitzer affirme avec perspicacité:

«L'espace ‘off`, le champ aveugle, c'est tout ce qui grouille au-dehors ou sous la surface des choses, comme le requin des Dents de la mer. Si nous «marchons `à de tels films, c'est parce que nous sommes pris, plus ou moins fortement, dans les tenailles de ces deux champs, in et off. Si le requin était tout le temps sur l'écran, ce serait très vite un animal domestique; ce qui fait peur, c'est qu'il ne soit pas là.» ${ }^{22}$

Cela dit, si le requin de Shark Jaws était toujours à l'écran du jeu d'arcade, il ne serait pas plus domestique parce qu'il poursuivrait sans relâche le plongeur sous l'eau à la recherche de poissons (plus de poissons pêchés équivalant à plus de points) pour le «manger» (à savoir, jeu d'arcade oblige, pour lui faire perdre du temps de jeu).

Mais dès l'instant où le joueur a pu se déplacer à gauche ou à droite dans un monde qui n'est pas refermé sur lui-même et contenu dans un seul écran comme Pac-Man (Namco, I980) où tous les fantômes sont visibles immédiatement, les concepteurs ont tiré le meilleur parti des six zones du champ aveugle: l'espace au-delà de chacun des quatre bords du cadre, l'espace derrière le décor, et l'espace derrière la caméra virtuelle. Ils ont utilisé les espaces à l'écran et au-delà de l'écran pour créer une tension autour de menaces mortelles. Car l'écran du jeu vidéo n'a pas plus de coulisses que celui du cinéma. Le champ aveugle vidéoludique est très loin d'être une zone morte. Au contraire, il constitue la plupart du temps cet espace où le joueur avance vers la mort ou d'où vient le danger. Les araignées géantes viennent attaquer de chaque côté de l'archer dans Forbidden Forest, et les dragons volants depuis l'arrière-plan. Dans une vue aérienne, les énormes insectes dans Ant Attack (Sandy White, I983) peuvent apparaître de tous les côtés puisque le personnage-joueur peut aussi se mouvoir vers le haut et vers le bas du cadre mobile. Avec des monstres volants comme les Reptiles qui attaquent des airs dans 
Silent Hill (Konami, I999), et les araignées tombant du plafond dans certaines chambres de Alone in the Dark: The New Nightmare (Darkworks, 200I), cette partie du champ aveugle a sans aucun doute un caractère inquiétant.

Dans les jeux effrayants à la troisième personne ${ }^{23}$, il y a un écart entre le point de vue et le point d'action. Il y a un certain espace tampon autour du protagoniste dans le cadre qui donne généralement au joueur un peu de temps pour voir l'attaque d'un monstre venant de derrière la caméra. En contrepartie, les monstres ont l'avantage de repérer et de se précipiter vers un personnage-joueur avant qu'ils n’apparaissent dans le champ de vision du joueur. L'écran du jeu vidéo est donc encore plus centripète que l'écran du film. Il suscite une peur vidéoludique basée sur la jouabilité, car il montre constamment un espace qui se prolonge dans un univers cauchemardesque et dans lequel un joueur doit s'immerger. En conséquence, l'ennemi est en effet «pratiquement» partout et tout le champ audiovisuel est dramatisé. Dans une étude sur la réaction des joueurs, Joel Windels a montré qu’«une tension accrue peut être créée par une atmosphère oppressante. Cela va maintenir le joueur engagé et, en définitive, lui faire craindre le pire. Les jeux qui maintiennent les joueurs engagés même lorsque les événements ne se produisent pas (en présentant une atmosphère stimulante) sont plus susceptibles d'effrayer les joueurs lorsque ces événements se produisent ${ }^{24}$. En outre, la présence virtuelle implique également que l'ennemi peut finalement ne pas être là, ou qu'un endroit qui était auparavant libre de menaces soit ensuite habité par des créatures létales.

En guise d'exemple de ces stratégies, les effets de surprise créés par les surgissements successifs à travers les fenêtres volant en éclats au début de Resident Evil sont efficaces parce que ces fenêtres situées sur le côté du cadre devraient constituer un bouclier contre une telle entrée soudaine. A partir de ce moment, le joueur sait que la menace est omniprésente dans le manoir de Spencer. Au niveau sonore, le gémissement des morts-vivants à l'extérieur du cadre rappelle souvent que l'espace hors champ n'est pas sécurisé. Les mouvements prédéterminés de la caméra virtuelle dans Resident Evil: Code Veronica (Capcom, 2000) changent constamment la relation entre ce qui est sur et hors de l'écran. Et il vaut la peine de citer une critique de Silent Hill, jeu qui
23 Nous pouvons tout autant formuler cette remarque face à une vue aérienne ou latérale montrant un personnage dans le cadre.

24 Joel Windels, «Scary Game Findings: A Study Of Horror Games And Their Players», Gamasutra.com, 7 septembre 20II, en ligne: www.gamasutra. com/view/feature/I34848/scary_game_ findings_a_study_of_.php?page=5. 
25 James Price, «Silent Hill», Official UK Playstation Magazine, $\mathrm{n}^{\circ} 48$, août I999, p. 88.

26 Tanya Krzywinska, «Hands-On Horror», dans Geoff King et Tanya Krzywinska (éd.), ScreenPlay. Cinema/ Videogames/Interfaces, Londres, Wallflower, 2002, p. 209. inclut des cadrages et des mouvements de caméra inhabituels pour continuellement donner au joueur un sentiment d'inquiétude:

«Dans quelques endroits, la caméra oscille d'un côté à l'autre pendant que vous courez. C’est vertigineux, désorientant et, lorsque cela est combiné à un accompagnement sonore stressant, c'est carrément alarmant. Dans d'autres lieux, la caméra peut soudainement coller à une vue fixe. Quand cela se produit, vous pensez souvent: «Que se passe-t-il maintenant? > et «Où est le monstre? >.» 25

En outre, le travail de la caméra de Silent Hill offre un moyen de distinguer une variation à partir de cette tactique d'épouvante.

D'abord, ce que je viens d'exposer représente une tactique qui repose sur un champ aveugle discursif, à savoir sur des éléments extradiégétiques comme le cadrage et les angles de prise de vue. Tel que l'a souligné Krzywinska à propos de l'imposition des différents angles de caméra dans Resident Evil 3: Nemesis (Capcom, I999), il s'agit de «dissimuler des informations visuelles et de créer un effet prononcé d'enfermement ${ }^{26}$. Cependant, le champ aveugle peut aussi être diégétique, notamment lorsque le champ de vision d'un joueur est partiellement (ou complètement) brouillé, ou encore bloqué par des «barrières» visuelles associées au monde du jeu. Pour se référer à une belle variation sur l'effet

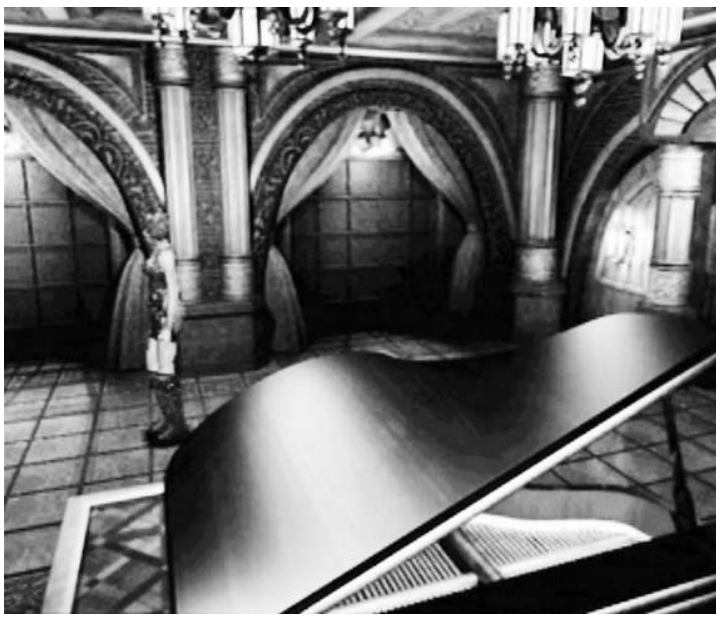

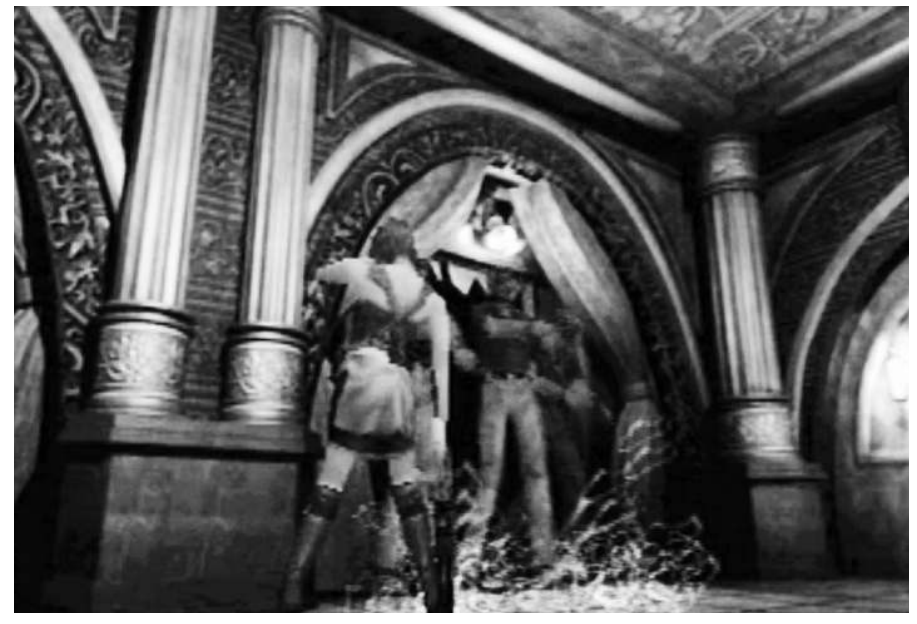


de surprise vu au début du premier opus, dans Resident Evil 3, Jill passe deux fois à travers une pièce avec un piano dans la «tour de l'horloge». Il y a en arrière-plan deux grandes fenêtres qui commencent au niveau du sol. S’il n'arrive rien la première fois, les zombies surgissent par lesdites fenêtres lors du second passage du personnage-joueur ( fig. I-2).

Lorsque les fenêtres sont très opaques, elles constituent pour les monstres de bonnes cachettes. Et selon le type de créature, les portes peuvent également servir de couverture ${ }^{27}$. En conséquence, cette possible irruption peut s'appliquer à n'importe quelle structure verticale, à l'image des murs de Project Zero (Tecmo, 200I) qui sont traversés par des fantômes. De même, avant de devenir volumétrique, le brouillard de Silent Hill n'était en fait qu'un mur gris, jamais atteint, mais devant lequel un chien pouvait être plus difficile à percevoir. Avec l'incertitude liée à la position des ennemis, la notion militaire de «brouillard de guerre» ( fog of war) couramment utilisée dans les jeux de stratégie en temps réel s'applique parfaitement ici.

Toutefois, le fait demeure que le champ aveugle diégétique le plus important dans l'horreur est sans aucun doute l'obscurité. Cela a été souligné plus d'une fois: c'est d'emblée effrayant d'être seul dans le noir. Dès Haunted House pour l'Atari 2600 (I98I), le joueur a dû explorer des endroits plongés dans l'obscurité totale. Sans les allumettes et le cercle autour des yeux du personnage-joueur, il était impossible de voir les pièces de l'urne qu'il devait chercher. Le jeu rétro à défilement latéral Home par Benjamin River (20II) s’inspire du même cadre circulaire incandescent durant l'exploration sans menace des lieux. Mais c'est une autre histoire quand un personnage-joueur doit s'appuyer sur une lampe de poche pour avancer dans un monde peuplé de nombreux monstres meurtriers qui se cachent dans le noir. Silent Hill a démontré à quel point il était effrayant de ne voir que ce qui est illuminé par un faisceau lumineux diffus alors que les monstres rôdent à l'extérieur de ce halo. Cette tactique de l'épouvante a été adoptée dans d'autres jeux comme The Ring: Terror's Realm (Asmik Ace Entertainment, 2000), Project Zero, Alone in the Dark: The New Nightmare et Alan Wake (Remedy Entertainment, 2010). Par exemple, lorsque Carnby pénètre dans le grenier ouest du manoir d'Alone in the Dark: The New Nightmare, alors qu'un Photosaurus - une créature reptilienne effrayée par la lumière - surgit du côté droit du cadre, un autre
27 Cela se produit dans le bloc d'appartements des Tours Cassini dans le chapitre trois de Dead Space 2 (Visceral Games, 20II). Quand Isaac s'approche d'une caisse devant une porte, un Necromorphe défonce soudainement cette dernière pour l'attaquer. 

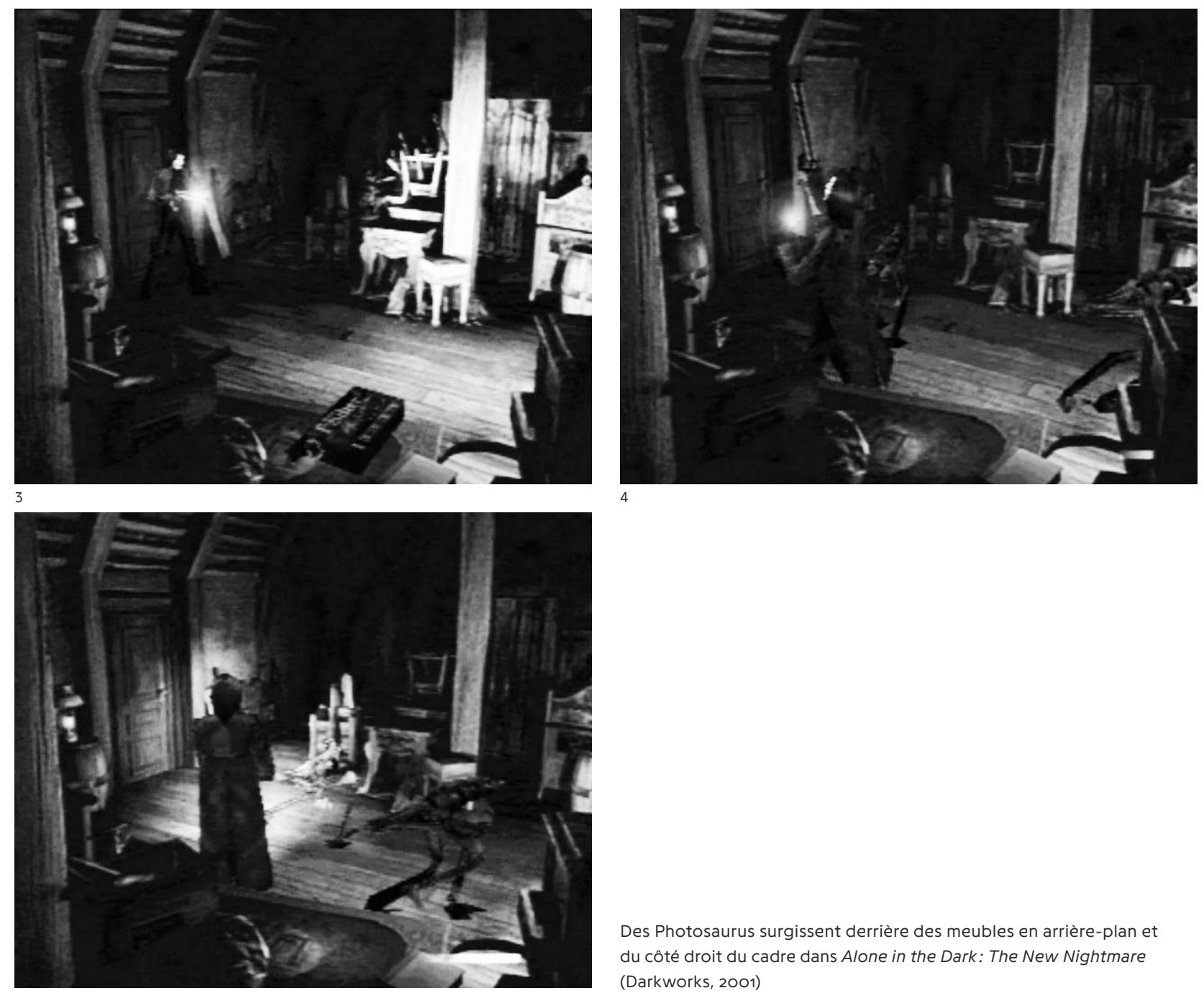

Des Photosaurus surgissent derrière des meubles en arrière-plan et du côté droit du cadre dans Alone in the Dark: The New Nightmare (Darkworks, 2001)

apparaît derrière des meubles dans la partie arrière plus sombre de la pièce (fig.3-5).

Le champ aveugle discursif créé par le cadre est de la sorte couplé à un champ aveugle diégétique. J'ai déjà décrit ce processus à propos de la lampe de poche dans Silent Hill: 
"Le halo de lumière crée un masque à l'intérieur du masque de l'écran de jeu. Cadre maîtrisable à l'intérieur du cadre de cet écran, son faisceau lumineux double l'effet de ce que l'on ne voit pas et ce qui se trouve hors champ. Il crée littéralement un jeu entre le visible et l'invisible, entre la lumière et l'obscurité (surtout avec un faible niveau de luminosité).» ${ }^{28}$

Les angles de caméra prédéterminés peuvent bien montrer le personnage-joueur dans un plan plus large, avec une certaine zone visible au-dessus et au-dessous de ce dernier, la vision du joueur n'est pas moins limitée à ce que la lampe de poche illumine.

Le régime effrayant de la vision à la troisième personne correspond à la description de Shaviro. S'il est réducteur d'associer l'expérience d'un jeu centrée sur un protagoniste à une approche transpersonnelle de l'existence humaine qui intègre des dimensions spirituelle, transcendante, sociale et communautaire, cette vision reste sans aucun doute «ni précisément objective ni conventionnellement subjective». Les angles de caméra et les mouvements prédéterminés du survival horror ont été choisis par le concepteur (tout comme au cinéma); cela dit, le joueur a toujours le loisir de se déplacer dans l'espace, de changer et de choisir par/ pour lui-même la façon dont l'action sera cadrée dans ces limites imposées. Par ailleurs, pour revenir à la catégorisation de Nietzel, le point de vue devient semi-subjectif lorsque la caméra suit un personnage comme dans Blue Stinger (Climax Graphics, 1999) et Resident Evil 4 (Capcom, 2005). Ces exemples ne correspondent pas tout à fait au point de vue (subjectif) du personnage-joueur, car un joueur voit (objectivement) le personnage dans le cadre, mais la vision est centrée sur et autour de ce point de (ré)action. Alors que la caméra est constamment dirigée vers un protagoniste et se trouve derrière lui pour présenter une vue «derrière l'épaule» [above-the-shoulder view], le joueur a la possibilité avec le balai droit de sa manette de changer le cadrage. La caméra peut par exemple tourner pour regarder derrière un personnage ou être ajustée pour avoir un meilleur angle pendant un combat. Certains jeux comme Silent Hill 2 (Konami, 200I) et le remake de Resident Evil pour la Nintendo GameCube (Capcom, 2002) ont une option de virage rapide à $\mathrm{I} 8$ o degrés afin de changer la direction du point de vue et du point de (ré)action. Les différentes vues de la caméra peuvent également traduire la vision subjective d'un personnage. Dans Cold Fear (Darkworks, 2005), le plan audessus de l'épaule de Tom Hansen (un garde-côte qui explore un bateau
28 Bernard Perron, Silent Hill: le moteur de la terreur, Paris, Questions Théoriques, 2016 [2006], p. I29. 
29 Tanya Krzywinska, «Hands-On Horror», op. cit., p. 209. russe en pleine tempête) concentre le regard du joueur sur le monstre lorsqu'il vise, tout comme l'est le personnage dans le monde du jeu.

Les sensations somatiques de ce régime de vision sont liées au corps à l'écran, au corps spéculaire d'un personnage-joueur. Un joueur contrôle ce dernier, mais il regarde toujours un protagoniste à l'écran. La subjectivité de ses actions et l'objectivité des mouvements du personnage restent difficiles à déterminer. Il n'en demeure pas moins que le joueur est très certainement plus frappé par l'horreur quand il voit son personnage être tué à l'écran. La visualisation des personnages permet d'introduire un ensemble d'effets «gore». Forbidden Forest peut être défini dès I983 comme un «video game nasty» (une référence aux films violents distribués sur cassette vidéo dans les années i980 en Angleterre) et provoquer des réactions puissantes parce que l'archer meurt de manière horrible. Le sang gicle aussi avec quelques pixels dans l'adaptation d'Halloween la même année et à grands jets lorsque Jill et Chris sont mordus par un zombie dans Resident Evil.

\section{Le régime effrayant de la vision à la première personne}

Conformément à l'analyse comparative des modes à la troisième personne de Resident Evil 3: Nemesis et à la première personne de Clive Barker's Undying (DreamWorks Interactive, 200I) effectuée par Krzywinska, ce dernier mode confère au joueur une vision à 360 degrés lui permettant de regarder où il le souhaite et l'encourageant à étudier l'environnement en détail puisqu'il peut dès lors le balayer librement. Krzywinska note surtout que «[] a liberté de regarder et d'explorer dans Undying marque une rupture avec la façon dont les films d'horreur utilisent le montage et le cadrage pour créer de la tension et de la claustrophobie» ${ }^{29}$. Ainsi, d'une part, le concepteur de jeu ne peut pas utiliser directement de telles techniques cinématographiques pour créer des effets de suspense ou de choc. D'autre part, le joueur n'est plus soumis à des angles ou à des mouvements de caméra prédéterminés; de plus, il n'est pas non plus limité par la distance imposée par la caméra, qu’elle soit arrimée derrière le personnage ou qu'elle tourne autour ce celui-ci. Mais il y a un inconvénient - ou inversement un avantage, selon la façon dont nous voyons les choses - à cette option.

Dans une description du point de vue à la première personne en lien avec la question de l'identification et de l'immersion, Frans Mäyrä affirme: 
«Le joueur a la forte sensation d'être là, aucun personnage médiateur n'étant porté au centre de l'attention. Quand il est nécessaire d'inspecter un lieu où des monstres sont potentiellement embusqués, le joueur doit faire le mouvement lui-même - il n'y a pas d'autre position de caméra qui laisserait une marge de manœuvre. Le joueur peut seulement voir ce que le protagoniste du jeu peut voir.» ${ }^{30}$

Le joueur est rapproché de l'environnement, sinon plongé dans l'environnement, comme c'est le cas pour la réalité virtuelle (j’aborderai l'expérience de la réalité virtuelle dans la dernière section de cet article). Cette proximité n'est pas seulement une question d'espace, comme le souligne Krzywinska:

«Dans Undying, la forme particulière de restriction fournie par le mode à la première personne renforce l'illusion que c'est le joueur, assis devant l'écran, qui est attaqué, plutôt qu'un moi virtuel abstrait. Quand un monstre frappe directement l'avatar, par exemple, le cadre tremble et le viseur du joueur devient instable - le champ visuel du joueur et sa capacité à agir sont directement affectés par les coups.» ${ }^{31}$

L'observation de Krzywinska résonne avec le commentaire de Shaviro sur les séquences de SQUID dans Strange Days:

« Notre identification à la caméra, ou à la scène, est entièrement séparée de toute identification avec le personnage. Nous sommes attirés puissamment dans l'action, mais pas dans l'esprit de la personne qui a réalisé l'action.» ${ }^{32}$

Peu importe le personnage-joueur, qu'il soit identifié ou non, que son corps soit montré ou non dans une cinématique, c'est le joueur qui est «puissamment» plongé dans l'action d'un jeu d'épouvante à la première personne.

Dans ce régime effrayant de la vision, et cela est fondamental, le point de vue et le point d'action se chevauchent, rendant le personnagejoueur invisible à l'écran. C'est ce qui renforce l'illusion suivant laquelle la personne qui joue est aussi celle qui agit. Le regard étant en mouvement, il peut aisément être lié, comme chez Shaviro, à la notion de vision tactile. Mobilisant le rythme des actions du joueur réalisées à l'aide d'une manette ou de périphériques de contrôle, et les mouvements diégétiques au sein du monde du jeu, le jeu vidéo joue d'autant plus sur «les facultés 〈haptiques〉 de l'œil, en sus de la dimension 〈optique〉 plus commune» ${ }^{33}$. Le point de vue peut se rapprocher volontairement pour «toucher avec
30 Frans Mäyrä, Introduction to Games Studies. Games in Culture, Londres, Sage Publications, 2008, p. I07.

31 Tanya Krzywinska, «Hands-On Horror», op. cit., pp. 214-215. Alors que les actes de violence peuvent être dirigés contre le corps d'un personnage, c'est en effet le regard du joueur qui est assailli, l'écran clignotant habituellement en rouge à chaque coup.

32 Steven Shaviro, "Regimes of Vision: Kathryn Bigelow, Strange Days», op.cit., p. 63.

33 Thomas Elsaesser et Malte Hagener, Film Theory. An Introduction Through the Senses, New York, Routledge, 20Iо, p. Iо. 
34 Pensons à la façon dont on perçoit la texture des murs ou des objets dans un jeu vidéo quand on s'en approche très près.

35 Comme elle l'écrit, «les films d'horreur s'intéressent de manière obsessionnelle à l'idée selon laquelle le simple fait de regarder peut terrifier, mutiler ou tuer son objet - qu'un regard dur et un pénis dur (scie à chaîne, couteau à perceuse) équivalent à une seule et même chose» (Carol J. Clover, Men, Women and Chain Saws: Gender in Modern Horror Film, Princeton, Princeton University Press, I992, p. I82). Par conséquent, « regarder par l'entremise d'une caméra à la première personne fait partie de l'apanage de l'horreur» (id., p. I83).

36 Kathrin Fahlenbrach, "Affective Spaces and Audiovisual: Metaphors in Video Games», dans Bernard Perron et Felix Schröter (éd.), Video Games and the Mind. Essays on Cognition, Affect and Emotion, Jefferson, McFarland, 20ı6, p. I44. les yeux» ${ }^{34}$. En retour, il donne aussi le sentiment d'être touché quand, en tant que point de (ré)action, il est signifié - entre autres par l'entremise d'un scintillement rouge - que le personnage est atteint pas un projectile ou frappé par une arme blanche. D’ailleurs, comparativement au cinéma d'horreur, où, comme le note Carol J. Clover à partir des slashers des années i980, le regard est «assaillant» ${ }^{35}$ et demeure celui du monstre, le jeu vidéo représente principalement la perspective à la première personne de personnages humains. Et il met en scène un scénario commun que nous pouvons appeler, en suivant Kathrin Fahlenbrach, le schéma «chasseur-proie»:

«Les joueurs sont mis dans des situations paradigmatiques de combat, de meurtre, de destruction - ou de fuite, de camouflage et d'évitement d'un antagoniste. Le joueur active la connaissance paradigmatique des émotions, pour évaluer et réagir par réflexe à une situation donnée en développant des stratégies d’adaptation adéquates. En conséquence, l'évaluation réflexive par valences affectives guide de manière pertinente le comportement et les mouvements des joueurs.» ${ }^{36}$

Contrairement aux jeux de tir à la première personne qui donnent à un joueur toute la puissance de feu nécessaire afin d'aborder les différentes situations conflictuelles en tant que chasseur, ciblant l'ennemi et explorant le monde du jeu avec un regard assaillant, les jeux vidéo d'épouvante placent la personne qui joue en position de proie, balayant l'espace avec un regard précautionneux et anxieux. C'est exactement ce que visait Malcolm Evans lorsqu'il a conçu 3D Monster Maze (I98I) en plongeant le joueur dans un labyrinthe: le T. Rex est le chasseur et le joueur la proie. Aussi avec des dinosaures, la jaquette arrière de Dino Crisis (Capcom, I999) annonçait que l'équation n’avait pas changé quinze ans plus tard: «Intelligence artificielle prédatrice avancée... Etes-vous le chasseur ou la proie?». Des jeux tels que Penumbra: Black Plague (Frictional Games, 2008), Amnesia: The Dark Descent (Frictional Games, 2010) ou Slender: The Eight Pages (Mark J. Hadley, 2012) qui ne fournissent aucun moyen de défense (une autre tactique importante de l'épouvante) exacerbent une situation déjà problématique, ne laissant d'autre choix que de fuir. Loin d'être prédateur, le regard peut même devenir précaire. Dans Slender: The Eight Pages, un joueur ne peut pas scruter le champ aveugle puisqu'observer le grand homme mince l'amènera à se rapprocher plus rapidement et à venir mettre fin à la partie. L'un des conseils donnés dans 
Amnesia: The Dark Descent est d'«éviter de regarder les ennemis trop longtemps. Cela affectera votre santé mentale et vous serez finalement vu» (fig. 6). Quand, caché dans l'obscurité, Daniel regarde un monstre trop longtemps, l'image devient brumeuse et ce dernier finit par s'évanouir pendant un instant.

Parce que les armes de mêlée sont fragiles, les armes à feu rares et les munitions limitées, Condemned: Criminal Origins met en avant le fait qu'il s'agit de se défendre et de rester en vie avec les moyens disponibles plutôt que de tirer sur tout ce qui bouge. Et, s'il y a des moments où un joueur peut devenir chasseur quand il sait qu'un ennemi se cache, son activité de poursuite et de mise à mort est le plus souvent conditionnée à une précaution pour enquêter sans danger dans une zone et empêcher une future escarmouche. 
37 Tanya Krzywinska, «Hands-On Horror», op. cit., p. 213.

$38 \mathrm{La}$ série est connue en Amérique du Nord sous le titre de Fatal Frame.
Comme le souligne Mäyrä, un joueur n'a accès qu'à ce que le protagoniste peut voir dans la perspective à la première personne. Cependant, la vision diégétique n’est pas nécessairement égale au large champ visuel dont on dispose dans la vie réelle. Krzywinska a à juste titre souligné que «les jeux peuvent bien donner au joueur la liberté de regarder, le cadrage à la première personne durant le jeu est, néanmoins, en termes cinématographiques, 〈restreint $\gg{ }^{37}$. Que ce soit à travers une caméra virtuelle ou les yeux du personnage (qui pourraient encore être envisagés comme un objectif de caméra), l'écran est toujours, selon les termes de Bazin, un masque ne permettant de voir qu'une partie de l'action.

Dans la mesure où elle permet au joueur de passer à volonté entre un point de vue à la troisième et à la première personne, la série Project Zero (Tecmo, 200I-20I5) ${ }^{38}$ expose très bien la différence entre les deux perspectives. En fait, passer du mode terrain (troisième personne) au mode viseur de la camera obscura (première personne) - cet appareil photo est en fait l'arme utilisée pour se défendre contre les fantômes - revient à passer d'un plan large à un plan rapproché ou à un gros plan, soit à entrer (cadre diégétique) et à sortir (cadre discursif) du monde du jeu (fig. 7 -8).

Du mode terrain (troisième personne) au mode viseur de la camera obscura (première personne) dans Project Zero II: Crimson Butterfly (Tecmo, 2003)
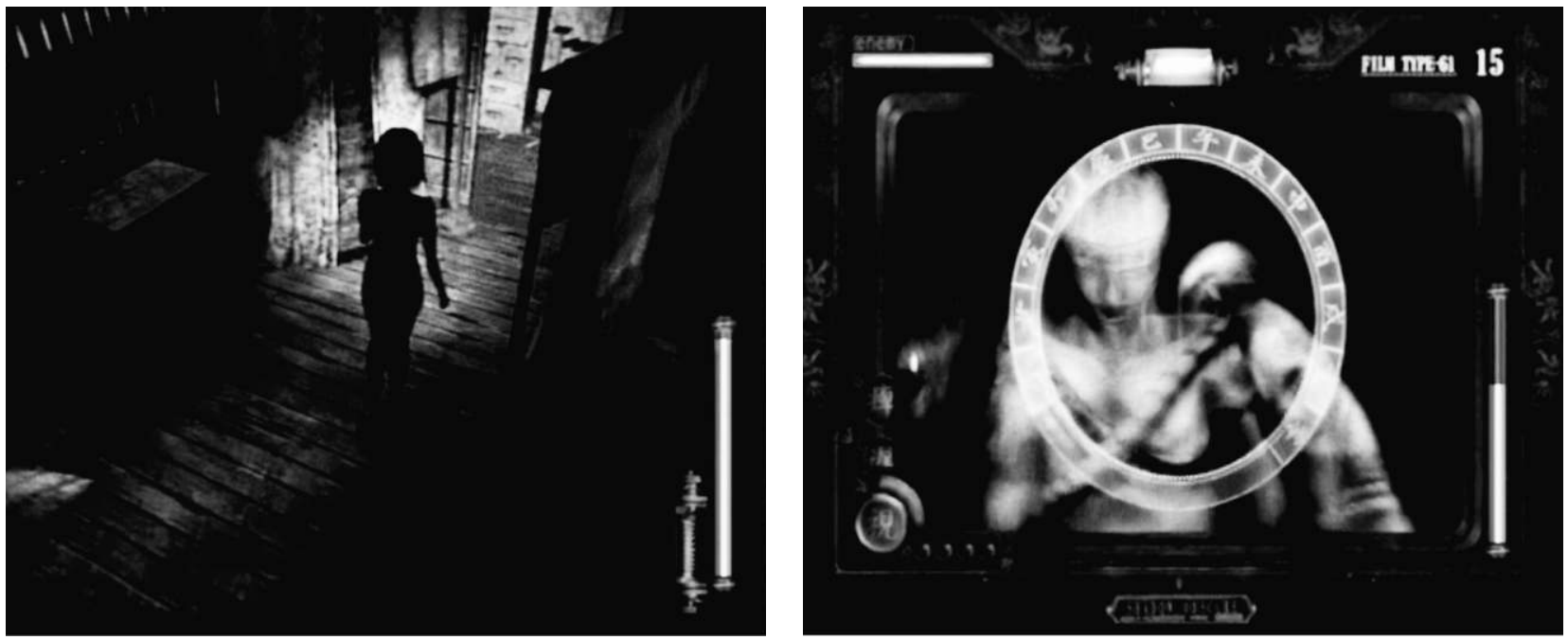
Il n'y a pas d'espace tampon entre le point de vue et le point de (ré)action dans le dernier mode et en tant que tel, ce type de point de vue est plus contraignant. De plus, comme la perspective à la première personne de Project Zero affiche le viseur de la camera obscura diégétique, elle révèle l'impact des dimensions du cadre, de la «fenêtre sur le monde», comme dirait Bazin. Si un joueur a un champ visuel plus large pour repérer un fantôme hostile venant attraper l'une des jeunes filles alors qu'il balaie avec la lampe de poche une pièce vue à partir d'un angle en plongée, il doit rapidement se déplacer pour voir le fantôme avec le vieil appareil photo. En mode viseur, il y a un cercle de capture. Pour pouvoir charger à son maximum le pouvoir spirituel utilisé pour se battre, un fantôme doit être dans le cercle. Quand l'un d'eux se précipite vers le joueur, ce dernier doit donc attendre que le cercle devienne rouge et clignote. Suivant le manuel d'instruction:

«Cela signale un moment d’opportunité spécial appelé le cadre fatal. Si vous prenez une photo à cet instant, votre adversaire sera immédiatement repoussé, subissant des dégâts considérables, et vous gagnerez un grand nombre de points.»

L'inconvénient de ce «cadre fatal» (d'où le titre alternatif: Fatal Frame), c'est qu'il ne peut se produire que lorsque le fantôme est très proche de la camera obscura: autrement dit, le joueur doit avoir des nerfs d'acier et attendre le plus longtemps possible avant de prendre une photo. En d'autres termes, il doit littéralement faire face à la crainte de voir un fantôme se rapprocher de lui.

Lorsque le champ aveugle est diégétique, les monstres peuvent se trouver tout juste au-dessus du protagoniste, ramper sous son nez (et celui du joueur), ou se trouver directement derrière lui. Dans le monde du jeu, tout peut facilement bloquer ou réduire la vue. En outre, la mécanique de jeu qui permet de se cacher n'aboutit à rien d'autre qu'à un point de vue très restreint et un point d'action immobile. Se déplacer à l'intérieur d'un casier dans Outlast (Red Barrels, 2013) ou Alien: Isolation (Creative Assembly, 20I4), c'est limiter l'espace visible aux petites ouvertures d'aération du casier. De même, la distance très courte à laquelle peut se trouver un monstre explique en grande partie l'efficacité des sursauts de peur du régime effrayant de la vision à la première personne ${ }^{39}$. L'obscurité reste un lieu de surprises. Par exemple, lors de l'exploration à la première personne de la maison de Layers of Fear (Bloober Team, 20I6),
39 D'un point de vue visuel, il n'est pas plus aisé de se protéger contre le surgissement rapide d'un objet au sein du cadre dans une perspective à la troisième personne. Cette réaction de sursaut est une tendance humaine innée. De surcroît, l'apparition envahit la plupart du temps tout le cadre. Un monstre se déplaçant à la vitesse de l'éclair vers un protagoniste équivaut souvent à l'écran à une coupe rapide vers un gros plan. C'est notamment la stratégie de Five Nights at Freddy's (Scott Cawthon, 20I4). 

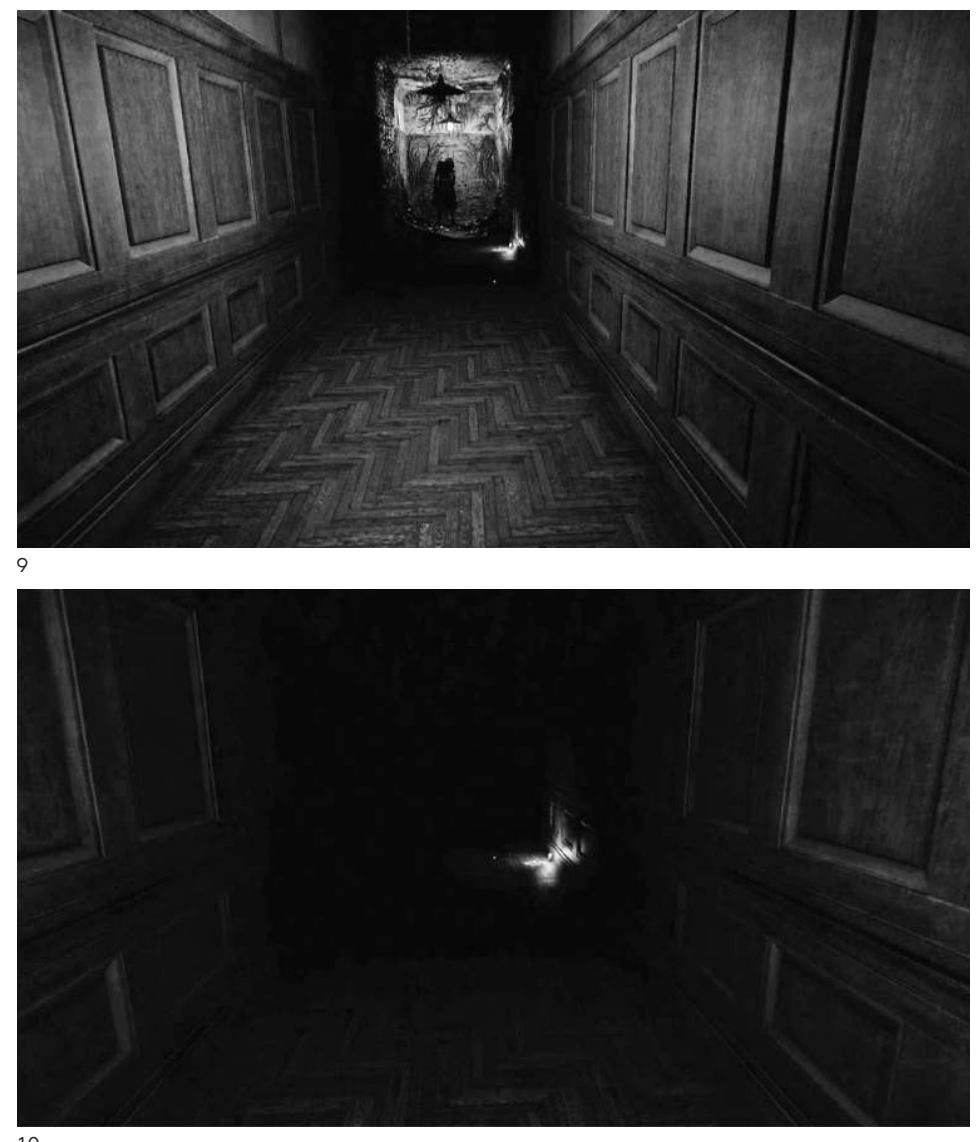

10

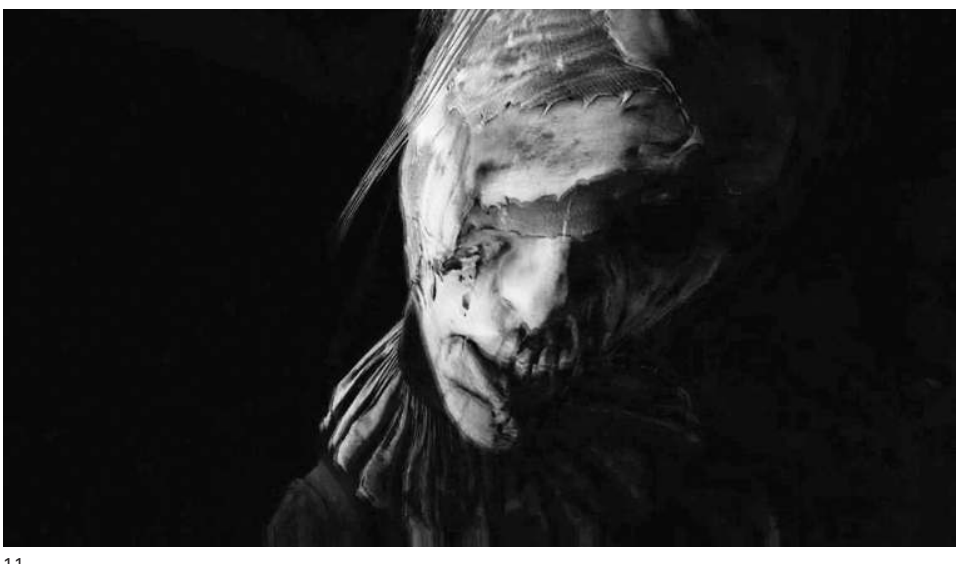


le personnage-joueur voit le fantôme d'une femme devant une porte au bout d'un couloir et entend un enfant pleurer. L'ampoule au-dessus de sa tête se brise, laissant l'arrière-plan noir. Quand le peintre s'avance et entre dans l'obscurité, un visage grisâtre surgit sur le côté inférieur gauche de l'image avec un fort bruit et le fantôme l'attrape (fig. 9-II) ${ }^{40}$. On le comprend à nouveau: tout peut surgir de chaque côté du cadre.

\section{Point d'interaction}

Il est difficile d'ignorer la réalité virtuelle lorsqu'il s'agit de définir le régime effrayant de la vision à la première personne. Commercialisée pour le grand public, celle-ci est en ce moment promue comme la nouvelle forme de divertissement au moyen de visiocasques (HDM) et de contrôleurs de mouvement. En outre, Resident Evil VII: biohazard (Capcom) a été au début de l'année 20I7 le premier jeu complet effrayant «triple-A» vendu pour la PlayStation VR. Suivant l'un des éléments centraux de l'essence de la réalité virtuelle, elle se voudrait plus immersive, car «le HDM coupe les sensations visuelles et sonores du monde environnant et les remplace par des sensations générées par ordinateur ${ }^{41}$. Une autre façon d'exprimer ces sensations est de renvoyer de nouveau à la notion de présence:

«L'important est que la réalité virtuelle vous donne l'impression d'être dans un lieu autre que celui où vous êtes vraiment [...]. Elle le fait en suivant vos mouvements corporels, vos mouvements de tête en particulier. Nos cerveaux semblent traiter le monde virtuel comme s'il était réel et notre corps réagit comme s'il l'était.» ${ }^{42}$

A partir des concepts développés par Neitzel, je soutiendrai que dans la réalité virtuelle, c'est le point d'interaction qui vient chevaucher le point de vue et le point d'action afin de casser le quatrième mur et brouiller la ligne entre le monde du jeu et l'espace extra-diégétique. Un joueur ne regarde pas un écran et ne se tient pas à distance de celui-ci. Dans la mesure où l'image épouse la représentation sphérique à 360 degrés, la personne qui joue est conduite à percevoir qu'elle interagit directement avec ledit monde du jeu. Le champ visuel tente alors d'être aussi proche que dans la vie réelle, répondant en effet aux mouvements de la tête ${ }^{43}$. La sensation d'être au niveau du sol vient du fait que, lorsqu'il regarde vers le bas, un joueur voit le terrain virtuel et le corps virtuel du protagoniste (s'il est modélisé) et non pas son propre corps ni le plancher de son
40 Il y a un épisode similaire dans P.T. (Kojima Productions, 20ı4). Au moment où le joueur tourne son regard diégétique vers l'entrée de la porte, le visage du fantôme Lisa est juste derrière lui. Elle l'empoigne, le secoue et le fait tomber par terre.

41 Michael Heim, The Metaphysics of Virtual Reality, New York, Oxford University Press, I993, p. II2.

42 Dan Staines, "Virtually Harmless?», Hyper, n 264, 20I6, p. 3I. Il cite en fait le $\mathrm{D}^{\mathrm{r}}$ Michael Madary de l'Université de Gutenberg.

43 L'une des premières publicités de la PlayStation VR met en scène un jeune homme avec la tête tournée vers la droite pour regarder avec effroi un dinosaure. 
En réalité virtuelle, l'eau monte jusqu'à la poitrine du (personnage-)joueur, le plafond est au-dessus de sa tête et un cadavre flotte près de son visage dans Resident Evil VII: biohazard (Capcom, 2017)

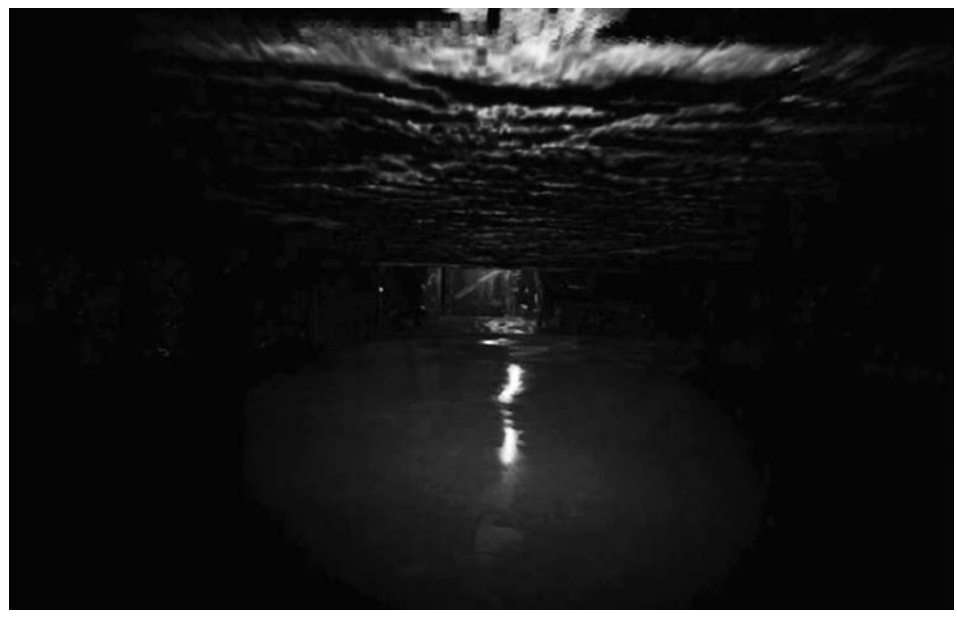

12

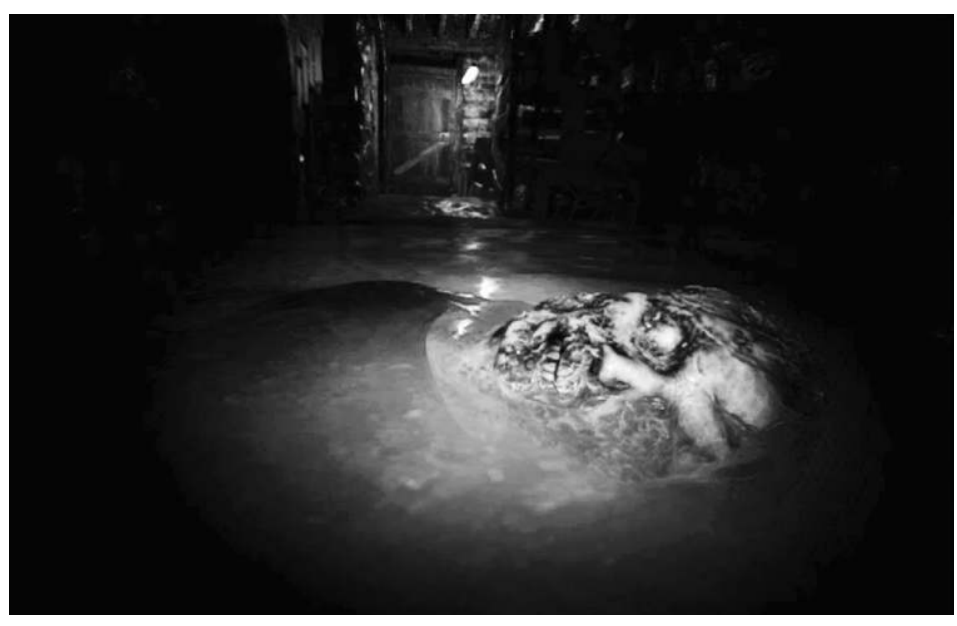

44 Il faut entre autres lever la tête pour voir le pont d'un immense navire dans Resident Evil 7: biohazard. salon. Cependant, en utilisant une manette, la PlayStation VR n'est pas si différente de la vue classique à la première personne. Tout est encore traduit à travers une vision tactile. Ce qui est alors plus frappant - et Resident Evil VII : biohazard en a pleinement profité - c'est qu'un joueur peut regarder vers le haut et voir l'espace au-dessus de lui ainsi que la hauteur des murs et des architectures ${ }^{44}$. Cela rend l'espace restreint encore plus susceptible de générer une peur intense et un sentiment désagréable 
d'être confiné dans des endroits étroits ou pris au piège dans une arène confinée. Par exemple, Ethan (le personnage-joueur de Resident Evil VII) doit parfois s'accroupir et marcher sous la maison des Barker; le plafond est alors près de la tête du joueur. Se mettre dans l'eau jusqu'à la poitrine fait aussi flotter un cadavre près du visage ce celui-ci (fig. 6). Quand un personnage non-joueur ou un monstre s'approche, la proximité se fait ressentir visuellement, donnant l'illusion qu'il se tient juste à côté du joueur. Sachant qu'une situation vidéoludique sera effrayante si elle est jugée dangereuse en elle-même, il est facile de comprendre que le haut degré de présence et l'implication émotionnelle accrue de la réalité virtuelle sont vraiment appropriés pour créer une expérience à glacer le sang, que Chloi Rad a définie comme «intense et personnelle» [up close and personal ${ }^{45}$.

\section{Voir, ce n'est pas tout}

L'objectif de cet article consistait à comprendre les différences et les similitudes entre le jeu vidéo et le cinéma afin de définir les deux principaux régimes effrayants de la vision dans les jeux vidéo d'épouvante. D’une part, la distance entre le point de vue et le point de (ré)action - et le point d'interaction dans la réalité virtuelle - modifie la façon dont l'aire de jeu est visualisée et explorée. D'autre part, qu'il s'agisse d'un point de vue à la première ou à la troisième personne, les deux perspectives reposent sur une forte sensation de présence et exploitent le champ aveugle discursif et/ou diégétique pour renforcer le sentiment suivant lequel le joueur n’est jamais en sécurité et continuellement traqué par des monstres. De plus, les effets de ces perspectives tiennent à une composante vitale: le son. Bien que j’aie parlé de vision et que l'on discute généralement de jeux vidéo sur le plan visuel, il ne faut pas oublier la dimension sonore. En référence aux travaux de Michel Chion, il serait plus juste de se référer aux jeux en tant que formes contemporaines d'audio-vision ${ }^{46}$. Jouer à des jeux d'épouvante, c'est faire une expérience auditive tout autant qu'une expérience visuelle. Il n'y a point de vision sans un point d'écoute. Et on le sait, entendre un bruit anormal sans connaître sa source, c'est toujours alarmant, surtout quand on est seul plongé dans le noir.
45 Chloi Rad, «Resident Evil 7: Biohazard Review», IGN.com, janvier 20I7, http://ca.ign.com/articles/20I7/or/23/ resident-evil-7-biohazard-review.

46 Michel Chion, L'Audio-vision, Paris, Nathan, I990. 